\title{
The Ecology of Education: A Social and Psychological Approach to Inequality
}

\author{
Ewa Sidorenko \\ University of Greenwich, United Kingdom
}

\begin{abstract}
In many discussions about persistent structural inequalities of educational outcomes in UK two important aspects are left out from analysis: the dominant philosophy of education (culture of learning), and psychological insight into educational 'failure'. The paradox is that without a nuanced approach to analyzing differential outcomes, conventionally, sociology of education has tended to treat learners as subject to structural processes of socialization in which they themselves had no agency. What the persistence of patterned educational 'failure' suggests, is that there are indeed processes at work generating such outcomes but these may be more complex than those underpinning ideas which claim that particular groups are 'failed by the educational system'. This paper argues for a complexity perspective approach which conceptualizes the school as a social ecology in which learners, as agency, are significant constituent elements in a dynamic relationship with the institutional framework of the school which includes both structural and cultural factors. This paper argues that we need to move the entire debate around failure away from the reductive focus on the 'school' or the 'system' without proper attention given to the complexity of the school ecology.
\end{abstract}

\section{Introduction}

Much of sociology of education and the wealth of critique of education have focused, over the years on the analysis of systemically generated inequalities in educational outcomes. Similarly, policy developments in the UK since the 1960s have tended to redress the issue of educational inequalities not in terms of individualised concern but according to socially patterned marginalisation: by class, gender, ethnicity or disability. This, from the point of view of the ethos of social democratic philosophy has been necessary to maximise the reality of citizenship and social justice. This paper suggests that it is time to move further beyond the current attempts. The paradox of the social democratic interventions in education so far is that they are beginning to resemble classical liberal ideas of justice in which access (to the suitably reformed learning environment) represents equality. However, what we continue to witness in this country is a stubborn reluctance of cultural tendencies: social mobility in terms of educational achievement is relatively low, as compared to other developed countries. [1] What we need to focus on now in order to effect better educational outcomes across the board, is not just through systemic improvements in terms of the fairness of the playing field but also at the level of the learning cultures which shape respective learning constituencies. Moreover, we also need to open up our sociological framework to psychological insights gained from contemporary learning theory in order to understand other factors in socially patterned underachievement. Controversial though this may be politically, the issue is inescapable if we look at the educational field holistically.

I begin this paper by returning to the classical social theoretical dichotomy of structure/agency in order to position debates so far. I then consider the role of agency in education and links that can be made between learners as agency and their position within dominant philosophy of education. The thesis here is that sociological accounts of educational failure cannot deliver comprehensive analysis without attending to all of the constituent parts of the school social ecology.

\section{Structure vs. Agency?}

In the classical dichotomous problematic of structure versus agency in social theory, school tends to be seen as structure, whereas learners are considered to represent agency. Interestingly, despite developments in developmental psychology [3], the recognition from the late 1970s within the sociology of education of the agency engagement in terms of resistance to schooling from the working class lads [2], and finally, despite the more recent developments within the sociology of childhood recognizing the active and interpretive character of children [3], the educational debate within academic literature tends to focus primarily on the role of structure: the school as an institutional setting, or 'the system'. Traditional 
perception of the social science paradigm, portraying learning as a one way process of socialization in which external structures/ ideologies/ values/ norms are internalized by groups and individuals, needs to be updated. This is not an issue of academic preferences. The problem with focusing only on one side of the issue is that the learning environment cannot be successfully modelled and conceptualized as if the character of the learner did not matter. Learning needs active agency, and active agency needs to be created or realized in and through its social ecology. What we do need to do then, is to examine the school as social ecology in which there are dynamic relationships between its numerous constituent parts. From the point of view of the complexity perspective for schooling to be effective, there needs to be a qualitative 'fit' between the institutions of education and various agencies operating within the social ecology. In accord with recent developments in cognitive theory and learning theory, this process may be constructively seen as auto-exo-referential [4]. This perspective is fundamentally ecological. Let us consider this proposition in relation to current assumptions about the role of 'parental involvement' in contemporary educational policy.

\section{Cultures of learning: the battle over cultural capital}

There is presumption about benefits of parental involvement which in turn involves a certain quality and quantity of cultural capital. Moreover, it has been argued that underperforming sections of the community 'would benefit most' from parental involvement in their children's education [5]. Indeed, parental involvement is encouraged by schools explicitly through home-school policies in which schools take the lead in outlining the channels of communication with parents, and in organising specific opportunities and programmes for enhancing community support for learning. Yet the very existence of school policies promoting parental involvement rests on tacit recognition that parental involvement cannot be taken for granted as it does depend on the pre-existence of a degree of cultural capital. Parental involvement thus, alongside with other factors can be examined in the context of wider social inequalities. Addressing this issue analytically leads to politically uncomfortable propositions. Given the well established concept of cultural capital, a corresponding idea of cultural deficit seems inseparable. In other words, the idea of 'deficit' is the other side of the coin of 'capital'. The idea of cultural deficit, however, brings with it, a visible degree of discomfort. As stated in the introduction, much intervention has been attempted at the school level, and more recently, through the Third Way initiatives delivered by the Labour government in UK (19972010) aimed at building up cultural capital, a more holistic approach has also been tried. Yet, support for the community level intervention initiatives is weak among the educational literature concerned with the pathologising consequences of the cultural deficit discourse [6]. Policies targeting the community rather than school have been criticized as not recognizing the value of working class or minority ethnic cultures and for their repressive potential. [6] So how can a qualitative (cultural) change take place in a chicken and egg dilemma: should intervention be levelled at the community ('parents') or at the level of the school ('system')? A politically comfortable response to the problematic of cultural capital is to reform the school setting in such a way that this cultural capital would cease to command advantage. This would require that schools should become culturally neutral ecologies where all types of pupils' cultural capital are equal. Alongside with the 'system', 'bad teachers' could also be singled out for their role in failing some students. How viable is this idea for examining and redressing 'failure' due to educational disadvantage?

Our current school-community ecology seems to present the following problematic options for resolving the issue of cultural capital inequality at the school gate. Given the inseparability of cultural capital/ cultural deficit relations, if schools need to rely upon parental support for children's education in a comprehensive sense, it may well have to be created first because the reality is that parental involvement, as an enactment of cultural capital cannot be assumed to be always already there. Instead, it needs to be developed (explicitly) for it to be effective. But who is to create it if there is 'cultural deficit'? These are highly political arguments. Should we charge schools (or the state?) with the responsibility for 'making' parents involved and ensuring this is positive? Conversely, should we assert that as parents are children's first educators, they are always able to deliver such involvement with minimal support (effort on the part of the school/state). Arguably however, this approach may only further divide the outcomes between educationally advantaged and disadvantaged children. Indeed, some of the Third Way initiatives channelled though primary schools as well as Children's Centres have already began actively targeting parts of the community by actively and creatively involving parents with learning through local initiatives in order to contribute to the creation of the cultural capital [7]. Yet this locally targeted process could also be enhanced by a more general approach in which a positive philosophy of education is promoted at a societal level. That would further 
empower schools to launch confident and creative programmes promoting parental involvement. I develop this point later on in this section.

The second possibility is to give up on the idea of parental involvement altogether; and let schools assume total responsibility for children's learning. Consciously or not, structurally speaking, this would be in order to make social background less ecologically significant in educational outcomes. Politically, in terms of social democratic expectations this would be preferable because then, children's social background would be less of a factor and there would not be the problem of the pathologising discourse. One practical way to achieve this would be to adjust expectations of all learners to correspond more to the learning abilities of slower students. In other words, slowing down the pace with which curriculum moves students along would enable weaker students to achieve better results. This principle underpins practices in other parts of the world where scholastic skills are introduced formally on average two years after English children's start of formal schooling. By the age of six, providing good quality pre-school is available, children's oracy skills and developmental levels are less diverse that those among four years olds [29]. Theoretically then, one way of reducing the advantage of children with stronger cultural capital input from their parents would be to slow the whole system down and pace the learning according to the lower (not the lowest) 'abilities'.

However, the pace of learning is not the only factor contributing to success or failure. Cultural capital consists of attitudes, motivation, emerging identity in relation to learning, desires for oneself and these are relative to learners' cultural background. Is it therefore possible, to design a system in which cultural advantage could be eliminated? What would that mean for the ecological position between the school and the community? Is that at all viable according to complexity perspective?

Removing the expectation of parental involvement and relying much less on community would mean regarding the school as a sui generis system with little or no input from its immediate locality. It is a proposal for insulation. But what would be the status of the pupils? Can their motivation and agency be created within the school gates? Or, should we accept the Marxist inspired perspectives, that schools and the external societal inequalities permanently and actively disengage young people from education; that this is their unavoidable function? Only through the most radical political reform of the relationships between the actual employment opportunities and education could we address this.

Let us consider the complexity of the school ecology in order to examine the possibility of reducing the influence of the community. The concept of social ecology enables a consideration of the learning (as well as teaching) processes within institutional settings as a set of inter-related systems. Just like natural ecology, social ecology is characterized by certain properties. First, the constituent systems are interrelated. Bad teaching is likely to result in poor learning (for at least some learners). Equally, challenging behaviour or poor motivation of learners might result in a cycle of negativity in the classroom, to a greater or lesser extent. Clearly then, creating classroom order and reaching particular educational aims by the teacher is an emergent property of the whole school ecology. In other words, the success of the educational process does not reside within the system (understood conventionally as an institutional agency of state organization) alone but is jointly accomplished through the combined inter-related processes taking place within particular space, time and with the dynamic interaction of relevant agencies. In natural systems, this means that order and optimal functioning need a stable supply of energy [8]. Clearly, it may seem a little odd to talk about energy in relation to human interaction yet, we know from the sociological tradition that this energy could be thought about in terms of cultural consensus (Durkheim) or domination (Marx) or authority (Weber, and, arguably, Foucault) or indeed as skilled and active accomplishment of social members (ethnomethodology). The point is that for order and learning to occur in the classroom, it is not just the structure in the form of institutional and material setting that needs to be in place. It is also the learner through their agency who needs to provide, or feed into the system the right input. In other words, the learner needs to be willing to accept the 'rules of the game'. What we are now finding from psychology, is that for some young people it is very difficult to supply a stable flow of engagement with learning, and motivated participation in the school setting, and hence the learning processes. That then means that the classroom ecology is composed of agency (or agencies) that are ontologically unstable, and hence, make this a volatile system.

What this shows is that even if the school abandons its more or less explicit expectations of parental involvement, the school ecology still needs the learner as agency to supply the right kind of input into the dynamic of the ecology. This also shows that we can never regard learners as blank slates, either sociologically or politically. It may well be possible to reduce the dis/advantage of particular parental background but only up to a point.

To elaborate this point let us consider Bourdieu's concept [9] of habitus, and its relationship to the field. For Bourdieu, habitus is ultimately a set of subjective dispositions which have been shaped by individual 
agents in relation to the field; the external yet immediate and meaningful world. For Bourdieu cultural capital, to a large extent, reflects the field in which individuals find themselves. Thus an important aspect of the school ecology, is the field with its supply of legitimacy of schooling, and the provision of values, philosophy and shared ideas towards the school. In other words the field can be seen as a cultural repository of attitudes and practices associated with schooling and school learning. If we apply Bourdieu's thinking to the learner, we can see that their intention and motivation are developed individually yet in response to their immediate field, i.e. cultural milieu. The, field, to continue with Bourdieu's concept is not an isolated micro-culture either. It may be local and it may be shaped by particular communities but has a recognisable pattered identity. Hence, it makes sense for sociologists to focus on 'white working class boys' or 'underachieving black boys'. It will be argued here, that the learning culture needs to be co-created by the 'centre' through a stronger philosophy of education.

What this highlights again, is the extent to which school ecology cannot be thought of as an isolated, closed system which is able to assume total responsibility for educational outcomes. We need think of the school ecology as including communities parents and neighbourhoods whose values, qualities and problems learners bring with them into the school. Therefore, we can enquire about the way in which interventions can be made more effectively also outside the school gate. Further, I suggest that one such intervention could be an attempt to address the issue of the legitimacy of schooling and the philosophy of education.

So far I have suggested that addressing the issue of 'cultural deficit' can lead to two alternatives: one, supporting the community in order to promote the desired cultural capital [9] or to try and isolate the school from the influence of cultural differences within the community. The first solution has been attempted by various interventions, including many by the New Labour government, yet has been criticized by academic literature for pathologising sections of the community [6]. The second solution seems attractive politically because it promises a reduction of cultural capital advantage but it would also need structural adjustments, to ensure that curriculum was delivered at a pace suitable for slower learners. However, as we see from the ecological perspective, the idea of school level intervention alone is not viable because the school is not an insulated but open ecology. The learner as agency is constituted both inside and outside the school and there needs to be a degree of cultural fit between their own selves and institutional framework for the ecology to be viable.

\section{Agency as habitus: psychological dynamics}

So far in this paper it has been argued that in order to provide an examination of dynamic interactions of various agencies within the school ecology we need to include the role of the field as co-constituting the learning agency. Before discussing possible interventions into the field, I now turn to a psychological explanation of some barriers to learning. This will help highlight the significance of the learner input in the school ecology in which some dynamic circumstances adversely affect the learning agency.

Illeris [14] lists three types of obstacles to intended learning: mislearning, defence and resistance. Mislearning is the least problematic form; it is a problem of error, of content. It results from mistakes or omission. This kind of problem can be easily rectified because there is no opposition to learning. Defence is an unconscious mental mechanism developed as a result of prior negative experiences, which is activated (unconsciously) in similar situations. In the context of learning, defence might lead to a denial of the problem, a dismissal of the challenge. This is a defence mechanism which protects individuals' mental balance, in the face of overwhelming information. According to Illeris [14] we need such mechanisms in order to protect our mental health. In a stronger version, defence is activated to protect identity. Identity defence helps agency to survive conditions which are perceived to be depriving them of control over their own lives. Defence then results in not learning, when learning is intended. '[T]the condition also exists, although in less massive forms, among many other young and adult participants who have not fully accepted that the education in which they are participating is suitable or necessary for them. In fact, they more or less experience it as 'having been placed' there by, for example the public employment service or the social authorities. Thus, identity defence can, in general, be characterised as the most profound and usually also the strongest defence mechanism against learning which, as a rule, is intended by others but not, or only partly, accepted by the person in question' [28] However, overcoming defence is possible only when agency has a 'strong core identity' [14], or 'ontological security' [15]. This, in some cases, might be an unreasonable expectation. The idea of core identity and ontological security will be developed in more detail in the next section.

Finally, Illeris identifies resistance as actively deployed by agency in the face of what is perceived as unreasonable demands or circumstances [14, can be experienced with significant levels of frustration. 
These conditions present as 'insurmountable obstacles that limit [...] life fulfilment' [16]. In children and young people resistance can present as difficult or challenging behaviour. Whilst resistance is often viewed by schools as negative and disruptive, Illeris [14] argues that 'it is often in connection to resistance, that the most important transcendent learning occurs. Personal development in particular, which is currently accorded such significance in education, often occurs through a process characterised by resistance' [17]. It can also be argued that expression of resistance or recognition of defence deployed by learners constitutes, what is considered by complexity theory, feedback which is crucially needed for systems to be able to adjust. In other words, if we really want learning to take place (as opposed to just keep groups of adolescents off the streets), we need to understand learners’ psychological challenges.

\section{Identity work and schooling}

What is clear then is that an ecological account of educational 'success' or 'failure' will need to go beyond a sociological perspective to include learners' psychological dynamics affecting social ecology of the school. Here, I would like to return to the social character of learning (or its failure) by linking the psychological factors to the issue of identity work. This will enable a psychologically informed account of socially patterned educational inequality, or in other words a social ecological account.

According to Illeris [14], learning needs to be looked at holistically and not just in terms of competence development but in the way in which the whole person of the learner interacts and changes through the process of learning: 'identity development can be understood as the individually specific essence of total learning' [18]. Erikson's concept of identity development [24] includes both social and individual aspects, and significantly, one which evolves incrementally throughout the entire lifespan, and one in which identity crises need to be resolved successfully otherwise they return at a later stage. Illeris draws on Stern's concept of the core self [19] and on Giddens' ontological security [15] as necessary for the learning process to be possible. What that means is that the very possibility of learning as transformation or development presupposes the existence of a 'core self' rather than an ever changing, fluid fragmented identity. Yet in agreement with much postmodern work on identity, Illeris adds that the current societal conditions have moved the goal of identity development from stable to flexible. What flexibility of the self means is a high degree of adaptability rather than an absence of coherence.
Thus, unlike under the conditions of modernity, today's society needs 'both a core identity and extreme flexibility, which must not have the nature of identity confusion but, rather, that of constant reconstruction' [20]. To develop this point in more detail Illeris turns to the work of another contemporary learning researcher, Kegan [21], who developed a detailed characterisation of complex lifelong transformations of orders of consciousness or personal development. Kegan [21] outlines five transformative steps, which represent progressively more complex organisation of thought. Following Piaget and Erickson, Keegan's idea of development is related to cognitive development yet it encompasses the entire consciousness. He goes beyond Erickson's characterisation of the adult state of consciousness to include the conditions of post-modernity. Much of Kegan's attention is focused on the challenges posed by contemporary Western cultures in relation to adolescents in reaching step three (out of five) and in showing that despite routine expectations, some of these demands cannot be met by young people before the end of their adolescence (age twenty). Indeed, Illeris subsequently confirms that many people in contemporary society may struggle to reach step four (out of five) in which 'one can oneself control the more abstract matters such as generalisations, values, relations with others, role consciousness and selfawareness, and in this [step] one is controlled by abstract systems such as ideologies, institutions and identity' [22].

There is no space here to develop in any detail Kegan's developmental characterisation but it is relevant to draw attention to a couple of important points. The different states of consciousness as described by Kegan represent not just the chronology of the developing individual but also a characteristic of different historical periods and their principle of subjectivity. Thus Illeris [14] points out what would have been the final phase of personality development in Western Europe prior to the Enlightenment and the emergence of capitalism, step three is now considered to be 'traditionalism' and is an accomplishment expected of adolescents. Modernity would have required a further accomplishment, step four characterised by self-authorship whilst the conditions of post-modernity place yet another challenge; that of self-transformation.

We can see from this that our contemporary society demands more and more challenging accomplishments of identity development. Indeed, Kegan's [21] work outlining the progressively more complex orders of consciousness is subtitled The Mental Demands of Modern Life. Let us consider some implications from that. From many writers we learn that contemporary goals (of adulthood) are flexibility and the ability to re- 
shape and re-construct our lives when situation demands [21],[23] [15], [14]. However, according to Illeris, most people today struggle to reach the penultimate step in identity development; and this appears significant given the expectations of lifelong learning society. This is an important observation by Illeris given that postmodern writers tend to equate the cultural expectation with the accomplishment. What the reality might actually point to is that as more and more people have no choice but to live with uncertainty, those who have reached the fifth order of consciousness are in far better position to meet the 'mental demands of modern life' [21]. Is it possible that we could examine a social patterning of accomplishments of orders of consciousness?

Let us return to the context of schooling; a setting for the adolescent identity work and the expectations placed by our culture of young people developing the third order of consciousness. In cases where identity defence or resistance takes place it may be relevant to consider the specific barriers to learning as linked to identity work. Is it possible to speculate whether in extreme cases young people have not quite reached the second order of consciousness? Kegan [21] argues in a manner which is similar to Erickson that consciousness transformation is a process of overcoming challenges, which can be though about as crises. Whilst these challenges are crucial for the very possibility of development, Kegan [21] emphasises the importance of providing support, a bridge, as he calls it, for successful development. Indeed, Erickson argues that each crisis needs to be successfully resolved. Kegan [21] asks whether our culture provides the necessary support to adolescents in particular, having set them difficult challenges.

This question seems to be highly relevant in the context of examination of the school ecology. Psychological support needed to overcome complex challenges facing adolescents identity development is perhaps what the sociology of education has failed to appreciate, as an important factor in delivering the desired outcomes. It may therefore be appropriate, to enquire about psychological processes involved in identity development of those young people who struggle in contemporary school settings; who do not provide the degree of motivation (agency input mentioned earlier) needed to engage in learning. We may need to develop psychologically sensitive class analysis of the identity development, as identity work is part of the process of learning.

This proposition takes risks, as once again, it can be seen as focusing on the deficit albeit of psychological nature. Yet, let us not forget, that from the complexity perspective, the issue cannot be reduced to one aspect of the dynamic; it will also involve the examination of the 'system' i.e. the institutional context in which learners find themselves. This framework therefore does not need to 'blame' the community, its field and the student for not supplying the right learning identity (or agency); rather this approach will be able to comment on the approach that these students need, given their developmental characteristics. Given our society's recognition of the reality of change, and need for flexibility, why would we not want to question the appropriateness of the institutional setting developed, after all, under the societal conditions of deference in Victorian times?

Finally, let us consider what the post-modern demand of developing flexibility might entail in terms of socially patterned inequality in the UK. In order to be in a position to open oneself to the 'layers of extreme flexibility' [14] first a solid core needs to be in place. But how can young people develop the solid core under the material and cultural conditions of severe uncertainty? Children and young people living in families which are described as stable in terms of employment, mental health, material conditions, and ideally, but perhaps not crucially, good educational parental background can reach the fourth step (modernism) through the influence of modelling behaviour taken from the parents. Whether they go far in education and whether they will develop the flexibility needed for the changing economic conditions in future may depend on contingency; the point is, they have a chance to reach the learning and identity developmental stage as demanded of them by their life circumstances.

On the other hand, when the starting point is a family or the immediate community which cannot offer a sense of stability (employment, economic, or health) it may be harder to reach the third level, the socialized mind, let alone achieve the further steps of 'selfregulation, self-formation, autonomy, individuation' as constituent of self-authorship in modern identities. Moreover, if we read this process back to Erickson's stages of identity development, any unresolved crises from previous stages inhibit a successful resolution of the next [24]. Thus it may well be possible that preschool experiences may already result in difficulties in terms of behaviour expectations needed at even the primary level. According to Erikson [24] an inadequate or harsh care received already in the first year of life may well result in a low level of trust in the world, and in others which can negatively affect relationships with people, and hence, learning.

Thus, when the starting point for identity development in the context of educational setting is a socially disadvantaged background where stability of various kinds is not the basis for underpinning childhood experiences, the trajectory of development seems particularly uncertain. Not having role models in terms of qualifications or job identity; and thus not 
significant others engaged in successful life strategies generating stability may affect availability of life choices and so challenge for the adolescent identity work. One could hypothesise that in Kegan's terms, young people from socially deprived backgrounds may well experience a trajectory which propels them towards uncertainty (postmodernism) without having grounded their identity development in the more stable ideas of step four (modernism) or even, in extreme cases, in step three (the socialized mind). In other words, from a degree of insecurity, these adolescents perceive the world of uncertainty which demands of them flexibility for which they are not ready. Perhaps it may be possible to deploy Illeris' barriers to learning analysis to identity development, and suggest that in the face of the uncertain world you can have young people adopting defence and resistance mechanisms against identity work itself, which is constituent of learning [14].

\section{Education philosophy and the culture of learning}

The above suggests that in cases of severe cases of social disadvantage when young people due to their life circumstances have not been able to reach the necessary step of identity development, defence and resistance to schooling is psychologically understandable. Given much evidence from recent literature on resistance to schooling by disaffected sections of young people [10], it may be useful to enquire whether their field provides a meaningful enough discourse of learning for young people and whether the field itself could become the object of intervention. Resistance, as explained by Illeris is a conscious mobilization by the learner against a perception of unreasonable demands. Can we not consider intervention addressing both of the solutions discussed at the beginning of this paper which would, the idea of promotion of philosophy of education in order to legitimize schooling and help develop agencyas-learner, as opposed to agency-as-resistance?

Intervention thus could be thought of in cultural terms (in addition to other necessary types, such as actual support for particular communities). Inevitably, both locally and nationally targeted promotion of the idea of a national philosophy of education, as, for instance advocated by Alexander [11] would constitute a major change in the political philosophy of education towards a 'centre'. Is this acceptable given the importance of our class and ethnic plurality? The answer could be affirmative if we agreed that the value of education can be thought of in the same way as we think of values underpinning ideas of democracy; for instance, the notion of tolerance, equality, mutuality, respect etc. If we invest education with this kind of societal value in which it becomes a social good; a true form of cultural capital, to be shared out within society then we surely could invest the state, in the manner resembling the French Republic's attitude towards the value of education in respect of citizenship, with the responsibility of shaping as well as managing the process [12]. In other words, if we think of education as something which has a value in itself, a form of human enrichment which also serves the purpose of civilizing the community (in the sense developed by Elias [13]) then the 'centre' could be legitimately put in charge of co-constructing such philosophy. At present, we, in the UK still regard the role of the state with discomfort and hence allow a degree of abnegation of responsibility for the content of learning to the changing needs of the labour market. In other words, to put in Marxist terms, it is the capitalist economy which is, in the last analysis, in charge of the significant amount of the content of education if its goals and aims are understood in terms of learners' future employability. The state, in this philosophy is reduced to role of the traffic police - directing the flow of movement of policy and resources but never getting involved with defining the purpose, the goals or the destination. The weakness of the philosophy of education has been observed before [12]. Instead, the highly utilitarian and functional understanding of schooling as mainly concerned with producing employability in the context of the maverick globalized economy prevents enduring, even timeless messages about the value of learning being sent from the 'centre'. It also prevents the development of pedagogy [11], [12] which schools could rely on for their own localized legitimacy. For Alexander, 'pedagogy encompasses [the teaching act] together with the purposes, values, ideas, assumptions, theories and beliefs that inform, shape and seek to justify it' [26]. In this view the act and the method of teaching, what elsewhere referred to as 'the school' is considered to be contingent upon deeper cultural values or purpose, in a Durkheimian sense: the cultural consensus underpinning the school's raison d'etre. Alexander argues further: 'if pedagogy is the discourse that informs and justifies the act of teaching and the learning to which that teaching is directed, then substance, and justification in relation to a view of what it is to be educated, must precede judgement. Otherwise, it is hard to know by what criteria judgements of competence, success and failure in teaching can be applied' [27]. The question raised here, that of, the purpose of education has not, Alexander argues, been subject to a debate; instead Alexander considers the UK education as the 'hegemony of the culture of pragmatism and compliance' [28]. In other words, both teaching and 
learning has been dominated by politically driven ideas, aims and targets; these however cannot, without a stronger cultural philosophy is either legitimate or effective if they are not meaningful within the learners' fields, and not met with the corresponding cultural capital. What Alexander has been arguing for is for a debate about the purpose of school. This paper seeks to emphasise the analytical significance of the learning culture as one linking the school demands with the learning agency. The point made in this section is that a strong, explicit and confident philosophy of education which the state needs to take part in formulating is necessary to aid the development of legitimacy of schooling, its ethos within learners' field. This, as said before, is to ensure the viability of school ecology.

\section{Conclusion}

This paper has argued for the necessity of expanding our analytical lens to embrace the complexity of the school ecology. The social ecological perspective shows that effectiveness of the school cannot be assured without active and willing agency of the learners because, the school cannot be analytically treated as a closed system. One important consequence from this follows, that socially patterned educational inequality needs to be analysed using an ecological perspective which can and must attend to all significant factors. Analysis of such unequal outcomes cannot be productive if we reduce the complexity of the dynamics.

In this paper, I have argued that interventions into the promotion of development of cultural capital seem inevitable because the success of learning within school ecology depends on a certain qualitative 'fit' between its constituent parts. Acting on the school institutional framework alone cannot deliver the desired effect if the agency of the learner does not provide a level of cultural and psychological input (habitus) conducive to learning. The agency of the learner is hugely important both in terms of cultural capital but also in terms of processes involved in their identity work. It has been argued that unequal educational outcomes could be linked to socially patterned identity work, thus showing a psychological link between social disadvantage and underachievement.

\section{References}

[1] Blanden, J., Gregg P., Machin S. 'Intergenerational Mobility in Europe and North America', A Report Supported by the Sutton Trust April 2005.
[2] Willis P., 1977 Learning to Labour New York Columbia University Press.

[3] Corsaro, W., (1997) The Sociology of Childhood Thousand Oaks. California: Pine Forge Press.

[4] Smith, J. 'Modelling the Ecologies of Education Systems’, LICE 2010.

[5] Williams, B., Williams, J., Ullman A. 'Parental Involvement in Education' BMRB Social Research 2002.

[6] Gamarnikow, E and Green, A, (1999) 'The third way and social capital: education action zones and a new agenda for education, parents and community?' in International Studies in Sociology of Education vol 9 .

[7] Ball S., The Education Debate The Policy Press, Bristol 2008.

[8] Smith, J., and Jenks, C., (2006) Qualitative Complexity. Ecology, cognitive processes and the reemergence of structure in the post-humanist social theory London: Routledge.

[9] Bourdieu, P., (1990), Structures, habitus, practices. In P. Bourdieu, The logic of practice (Stanford, CA: Stanford University Press. Bourdieu, 1990.

[10] Evans, G., (2006) Educational failure and working class white children in Britain. Basingstoke: Palgrave Macmillan.

[11] Alexander, R., (2009), (ed) Children, Their World, Their Education. Final Report and Recommendations of the Cambridge Primary Review. Cambridge: Routlege.

[12] Alexander R., 2001, Culture and Pedagogy: international comparisons in primary education, Oxford and Boston: Blackwell.

[13] Elias, N., The Civilizing Process. Blackwell Publishing.

[14] Illeris, K., (1999) How We Learn. Learning and non-learning in school and beyond. Routledge: London

[15] Giddens, A, (1991) Modernity and Self Identity . Self and Society in the Late Modern Age. Stanford, California: Stanford University Press.

[16] (Illeris 2007:170).

[17] Illeris (2007: 172). 
[18] (Illeris 2007: 138).

[19] (Stern 1995 in Illeris).

[20] (Illeris 2007:142).

[21] Kegan R., (1994) In Over Our Heads. The Mental Demands of Modern Life, Cambridge, Massachusetts: Harvard University Press.

[22] (Illeris 2007: 148 after Keegan 1994).]

[23] Bauman Z., Liquid Modernity. Cambridge: Polity Press.

[24] Erikson E., Identity: Youth and Crisis Norton 1968.

[25] Alexander, R., (2008) Essays on Pedagogy.

[26] Alexander, R., (2008: 75).

[27] (Alexander, R., 2008: 47).

[28] Illeris 1999: 165-7).

[29] Rogers, S. et al, (2007), 'Ready for Reception? The advantages and disadvantages of single-point entry to school, Early Years, 27: 1, 47 - 63. 